\title{
A simple method to localise pleiotropic susceptibility loci using univariate linkage analyses of correlated traits
}

\author{
Manuel AR Ferreira ${ }^{*},{ }^{1}$, Peter M Visscher ${ }^{1}$, Nicholas G Martin ${ }^{1}$ and David L Duffy ${ }^{1}$ \\ ${ }^{1}$ Queensland Institute of Medical Research, Royal Brisbane Hospital, Brisbane, Australia
}

Univariate linkage analysis is used routinely to localise genes for human complex traits. Often, many traits are analysed but the significance of linkage for each trait is not corrected for multiple trait testing, which increases the experiment-wise type-I error rate. In addition, univariate analyses do not realise the full power provided by multivariate data sets. Multivariate linkage is the ideal solution but it is computationally intensive, so genome-wide analysis and evaluation of empirical significance are often prohibitive. We describe two simple methods that efficiently alleviate these caveats by combining $P$-values from multiple univariate linkage analyses. The first method estimates empirical pointwise and genome-wide significance between one trait and one marker when multiple traits have been tested. It is as robust as an appropriate Bonferroni adjustment, with the advantage that no assumptions are required about the number of independent tests performed. The second method estimates the significance of linkage between multiple traits and one marker and, therefore, it can be used to localise regions that harbour pleiotropic quantitative trait loci (QTL). We show that this method has greater power than individual univariate analyses to detect a pleiotropic QTL across different situations. In addition, when traits are moderately correlated and the QTL influences all traits, it can outperform formal multivariate VC analysis. This approach is computationally feasible for any number of traits and was not affected by the residual correlation between traits. We illustrate the utility of our approach with a genome scan of three asthma traits measured in families with a twin proband.

European Journal of Human Genetics (2006) 14, 953-962. doi:10.1038/sj.ejhg.5201646; published online 24 May 2006

Keywords: linkage; multiple traits; pleiotropic; multivariate; empirical; power

\section{Introduction}

Multiple traits are often tested in gene mapping studies with the expectation that this can identify both quantitative trait loci (QTL) that are specific for each trait and genetic pathways that are shared between correlated traits. The most common approach used for linkage analysis

*Correspondence: Dr MAR Ferreira, Queensland Institute of Medical Research, PO Royal Brisbane Hospital, 300 Herston Road, Brisbane, Queensland 4029, Australia.

Tel: + 6173845 3572; Fax: + 6173362 0101;

E-mail: manuelF@qimr.edu.au or Manuel.Ferreira@qimr.edu.au Received 23 September 2005; revised 6 February 2006; accepted 6 April 2006; published online 24 May 2006 of multivariate data is to analyse each trait individually and then compare the evidence for linkage at a particular position across traits. This approach has clear advantages: univariate linkage statistics can be chosen that are most adequate for each trait; typically, the analysis is not computationally intensive; and, as a result, estimation of empirical significance is feasible. However, in its simplest form, this approach has two major drawbacks: first, multiple trait testing increases the experiment-wise type-I error rate, a caveat often implicitly avoided. Second, it does not utilise the extra information provided by the crosstrait covariance intrinsic to multivariate datasets. An alternative approach is to combine multiple phenotypes into one or 
few composite scores that are then tested for linkage. ${ }^{1}$ Although this approach may provide an optimal score to detect major QTL shared between traits, it may be inefficient to localise QTL that are specific for particular traits or only shared by some but not others.

A more theoretically desirable approach for the analysis of multiple traits is to test for linkage between one marker and all traits simultaneously. The two most general methods currently available for this in human pedigrees are multivariate variance components (VC) and a modified Haseman-Elston (HE) method. Maximum-likelihood multivariate VC was long proposed. ${ }^{2-4}$ This approach not only models the covariance for each trait across relatives as a function of VC parameters, but it also models the covariance between traits for each individual and across relatives. Although this represents one of its major strengths, it can in some situations render this method computationally unfeasible, particularly when a large number of traits have been measured. Another important drawback of multivariate $\mathrm{VC}$ is that it does not always provide increased power when compared to the individual univariate analyses. ${ }^{5-8}$

A multivariate extension to the traditional HE method was proposed by Amos et $a l^{9}$ and evaluated more thoroughly by Amos et $a l^{8}$ and Allison et al. ${ }^{6}$ In contrast to multivariate $\mathrm{VC}$, this method is computationally efficient even when analysing a large number of traits, is robust to violations of normality and is applicable to selected samples. However, at present it cannot accommodate arbitrary pedigrees and provides lower power when compared to multivariate $\mathrm{VC}^{8}$

Hence, although methods such as multivariate VC and $\mathrm{HE}$ are theoretically desirable for the analysis of multiple traits, they can often prove unfeasible and/or inefficient. We have recently outlined an alternative robust approach for linkage analysis of multiple traits. ${ }^{10}$ This novel approach includes two related methods that combine results from multiple univariate linkage analyses to estimate the evidence for linkage between (i) one trait and one marker while correctly accounting for multiple trait testing and (ii) multiple traits and one marker as a test to localise regions that may harbour a pleiotropic QTL. Although the performance of both methods looked promising, it was not evaluated in detail. Therefore, the aim of this study was to describe in detail both methods and evaluate them empirically using a series of simulations.

\section{Methods}

We propose two methods to combine results from univariate linkage analyses of multiple traits for robust and powerful detection of human QTL. The Combinedcorrection method estimates how often the observed linkage between a trait and a marker would be expected by chance alone conditional on the marker characteristics (eg allele frequencies, informativeness), trait characteristics (eg heritability, kurtosis), total number of traits tested and correlation between traits. The Combined-sum method estimates how often the observed linkage of two or more traits to the same marker would be expected by chance alone, conditional on the marker and trait characteristics, total number of and correlation between traits.

\section{Combined-correction}

Consider that $m$ traits were individually tested for linkage to a trait locus $Q$ and that the $m$ observed linkage test statistics $T_{k}(Q)$ for $k=1,2, \ldots, m$ are stored in the $1 \times m$ vector $V(Q)=\left[T_{1}(Q), T_{2}(Q), \ldots, T_{m}(Q)\right]$. Any test statistics can be used and these can be different across traits. The Combined-correction approach estimates empirically a pointwise $P$-value for each test statistic $T_{k}(Q)$ that is corrected for multiple trait testing. The overall experiment-wise type-I error is correctly maintained at nominal levels. It involves five steps.

First, we test the same traits for linkage to $n$ marker loci simulated under the null hypothesis of no linkage and store the $m$ test statistics recorded with each marker $i$ in the vector $V\left(N u l_{i}\right)=\left[T_{1}\left(N u l_{i}\right), T_{2}\left(N u l_{i}\right), \ldots, T_{m}\left(N u l_{i}\right)\right]$, where $i=1,2, \ldots, n$. Second, we compute the empirical pointwise $P$-value of each test statistic $T_{k}(Q)$ as the proportion of $T_{k}(N u l l)$ statistics that were $\geq T_{k}(Q)$, and the empirical pointwise $P$-value of each test statistic $T_{k}\left(N u l l_{i}\right)$ as the proportion of $T_{k}(\mathrm{Null})$ statistics that were $\geq T_{k}\left(N u l_{i}\right)$. Third, we replace the test statistics of $V(Q)$ and of each $V\left(N_{u l l}\right)$ with the $-\log _{10}$ of the respective empirical $P$-value, so that $V(Q)=\left[-\log _{10} P_{1}(Q), \quad-\log _{10} P_{2}(Q), \ldots,-\log _{10} P_{m}(Q)\right]$ and $V\left(N_{u l l}\right)=\left[-\log _{10} P_{1}\left(N_{u l l}\right),-\log _{10} P_{2}\left(N u l l_{i}\right), \ldots,-\log _{10}\right.$ $\left.P_{m}\left(N u l_{i}\right)\right]$. Fourth, we sort the $-\log _{10} P$ within each vector, so that the highest $-\log _{10} P$ appears in position 1 and the lowest in position $m$. Note, and this is crucial, that the order of traits does not have to be the same in the ordered vectors $V(Q)$ and $V\left(N u l_{i}\right)$, a property that forms the basis for multiple trait testing correction. In the fifth and final step, we estimate the overall significance of each $-\log _{10} P_{k}(Q)$ as the proportion of $V(N u l l)$ vectors that have the highest $-\log _{10} P(N u l l) \geq-\log _{10} P_{k}(Q)$. This global pointwise $P$-value is corrected for multiple trait testing.

This approach is easily extended to estimate $P$-values that are also corrected for multiple marker testing, such as in genome scans. In this case, the requirement is that genome-scan replicates with the same number of markers, allele frequencies, missing data pattern and marker spacing have been simulated under the null hypothesis of no linkage and analysed with all $m$ traits. A $1 \times m$ vector $V(\mathrm{Null})$ of ordered $-\log _{10} P$ is then created for each marker of each genome-scan replicate as described above. The global genome-wide $P$-value of each $-\log _{10} P_{k}(Q)$ corresponds to the proportion of genome-scan replicates that 
have one or more $V($ Null $)$ vectors with the highest $-\log _{10} P($ Null $) \geq-\log _{10} P_{k}(Q)$.

\section{Combined-sum}

This method estimates the significance of linkage between a marker and multiple traits. The first four steps are shared with the Combined-correction approach (see above). In step five, for $V(Q)$ and for each of the $V\left(N_{u l l}\right)$ vectors, we calculate $m$ sum statistics defined by $S_{k}=\sum_{j=1}^{k}\left(-\log _{10} P_{j}\right)$; these are defined here as $S_{k}(Q)$ and $S_{k}\left(N_{u l l}\right)$, respectively, where $k=1,2, \ldots, m$ and $i=1,2, \ldots, n$. Similar sum statistics have been proposed to combine $P$-values obtained from the analysis of $m$ single-nucleotide polymorphisms. ${ }^{11,12}$ In the present case, these $m$ sum statistics are simply the cumulative $-\log _{10} P$ when considering the highest $-\log _{10} P$, the two highest $-\log _{10} P$, etc. In step six, we assess the pointwise $P$-value of each sum statistic $S_{k}(Q)$ as the proportion of $V(\mathrm{Null})$ vectors that have an $S_{k}(\mathrm{Null}) \geq S_{k}(\mathrm{Q})$, and the pointwise $P$-value of each sum statistic $S_{k}\left(\mathrm{Null}_{i}\right)$ as the proportion of $V(\mathrm{Null})$ vectors that have an $S_{k}($ Null $) \geq S_{k}\left(\right.$ Null $\left._{i}\right)$.

The significance of $S_{k}(Q)$ is expected to decrease as $k$ approaches the number of traits influenced by $Q$ (or by a pleiotropic QTL linked to $Q$ ) and to stabilise thereafter or even increase if $m$ is large and $k<<m .{ }^{11,12}$ If the significance of $S_{k}(Q)$ does not improve when compared to $S_{k-1}(Q)$, this suggests that there is no evidence for linkage between trait $k$ and marker $Q$ after accounting for the linkage between the $k-1$ traits and $Q$ and the correlation between all $k$ traits. Thus, the final two steps consist of identifying the $S_{k}(Q)$ that yields the smallest $P$-value and then assessing its overall significance while considering that multiple sum statistics were computed in the process. For this purpose, we adopt the simple permutation approach proposed by Hoh et al. ${ }^{11}$ First, in the seventh step, we identify the sum statistic $S_{k}(Q)$ with the minimum pointwise $P$-value and define this $k$ as $k_{\min }(Q)$, the sum statistic as $S_{k_{\min }}(Q)$ and the $P$-value as $P_{\min }(Q)$. If two or more $S_{k}(Q)$ statistics have the same $P_{\min }$, we take the $S_{k}(Q)$ for which $k$ is lowest. We then apply this same procedure to each of the $V\left(N_{u l l}\right)$ vectors to identify the $n S_{k_{\min }}\left(\mathrm{Null}_{i}\right)$ statistics and the corresponding $P$-values $P_{\min }\left(\mathrm{Null}_{i}\right)$.

Finally, in the eighth step, we estimate the overall significance of $P_{\min }(Q)$ as the proportion of $V(N u l l)$ vectors with a $P_{\min }(\mathrm{Null}) \leq P_{\min }(\mathrm{Q})$. This significance is defined here as $P_{\text {final }}(Q)$ and represents the global empirical pointwise significance for linkage between the $k_{\min }(Q)$ traits and the position being analysed, where $k_{\min }(Q)$ provides an estimate of the number of traits that are likely to be influenced by $Q$ or by a pleiotropic QTL linked to $Q$. We note that when $k_{\min }(Q)=1$, this approach is equivalent to the Combined-correction method, with the exception that it provides lower power as it corrects for the number of $S_{k}$ statistics computed. The Combined-sum approach can be also extended to estimate genome-wide significance as described above for the Combined-correction method. The steps that form both methods are summarised in Table 1.

\section{Simulations}

We simulated data for 250 sib-pairs under eight linkage models to compare the performance of the proposed methods with univariate and multivariate VC linkage analysis. For models 1-4, three traits were simulated with a total heritability of 0.75 , a genetic correlation between all traits of 0.33 and an overall phenotypic correlation of 0.25 . Alleles for an additive QTL with allele frequency 0.5 and in Hardy-Weinberg equilibrium were randomly assigned to parents; one maternal and one paternal allele was then randomly dropped for each of the two children. The proportion of variance explained by the QTL for traits 1,2 and 3 was, respectively, $0.25,0$ and 0 in model $1 ; 0.25,0.25$ and 0 for model 2 ; $0.25,0.25,0.25$ for model 3 ; and 0.25 , $0.12,0.06$ for model 4 . Residual polygenic effects were assigned from a normal distribution to maintain the total heritability at 0.75 and the total genetic correlation between traits at 0.33 . Models 5-8 preserved the same QTL and overall trait heritabilities as described for models $1-4$, but the genetic correlation between traits was increased to 0.66 , resulting in an overall phenotypic correlation of 0.50 .

Two additional models were considered that retained the same trait heritabilities but with no QTL effects on any of the three traits. The genetic correlation between traits was 0.33 (phenotypic correlation of 0.25 ) in model 9 and 0.66 (phenotypic correlation of 0.50 ) in model 10. These two models were used to assess the distribution of the test statistics under the null hypothesis of no linkage for models $1-4$ and models $5-8$, respectively (see below). For all models, we assumed no environmental covariance between traits.

One thousand replicates were generated for the linkage models $1-8$ and 5000 replicates for the null models 9 and 10. For each replicate, a fully informative marker in complete linkage with the QTL was then simulated with the program SIB-PAIR 0.99.9 (http://www.qimr.edu.au/ davidD/sib-pair.html) to test for linkage.

\section{Analyses of simulated data sets}

For each replicate of each model, univariate and multivariate VC linkage analyses of the three traits were performed with $\mathrm{Mx} 1.54 .{ }^{13}$ The multivariate model fitted was the same as that used to generate the three phenotypes (Figure 1) and the appropriate $\mathrm{Mx}$ script is available from http://www.psy.vu.nl/mxbib/ (script multilink1.mx). For both the univariate and multivariate analyses, a likelihood-ratio test statistic was computed as $2\left(\ln \left(L_{A E Q}\right)-\ln \left(L_{A E}\right)\right)$, where $\ln \left(L_{A E Q}\right)$ is the natural 
Table 1 Summary of steps that form the Combined-correction and Combined-sum approaches

\begin{tabular}{|c|c|c|}
\hline Steps & Combined-correction & Combined-sum \\
\hline 0 & \multicolumn{2}{|c|}{$\begin{array}{l}\text { Perform univariate linkage analysis of } m \text { traits with a measured genetic marker } Q \text {. Create } 1 \times m \text { vector } \\
V(Q) \text { with test statistics } T_{k}(Q) \text {, for } k=1,2, \ldots, m\end{array}$} \\
\hline 1 & \multicolumn{2}{|c|}{$\begin{array}{l}\text { Analyse the same } m \text { traits with } n \text { genetic markers simulated under the null hypothesis of no linkage. Create } 1 \times m \text { vectors } \\
V\left(N u l_{i}\right) \text { with test statistics } T_{k}\left(N u l_{i}\right) \text {, where } i=1,2, \ldots, n\end{array}$} \\
\hline 2 & \multicolumn{2}{|c|}{ Compute the empirical pointwise $P$-value of each test statistic $T_{k}(Q)$ and $T_{k}\left(N u l_{i}\right)$} \\
\hline 3 & \multicolumn{2}{|c|}{ Replace $T_{k}(Q)$ of $V(Q)$ and $T_{k}\left(N u l_{i}\right)$ of each $V\left(N u l_{i}\right)$ with the $-\log _{10}$ of the respective empirical $P$-value } \\
\hline 4 & \multicolumn{2}{|c|}{ Sort the $-\log _{10} P$ within $V(Q)$ and within each $V\left(N u l l_{i}\right)$, with the highest $-\log _{10} P$ in position 1} \\
\hline 5 & $\begin{array}{l}\text { Estimate the significance of each }-\log _{10} P_{k}(Q) \text { corrected for } \\
\text { multiple trait testing as the proportion of } \\
V(N u l l) \text { vectors that have the highest } \\
-\log _{10} P(\text { Null }) \geq-\log _{10} P_{k}(Q)\end{array}$ & $\begin{array}{l}\text { Calculate the sum statistics } \\
S_{k}(Q) \text { and } S_{k}\left(N u l l_{i}\right) \text {, defined by } S_{k}=\sum_{j=1}^{k}\left(-\log _{10} P_{j}\right)\end{array}$ \\
\hline 6 & - & $\begin{array}{l}\text { Assess the pointwise } P \text {-value of each } \\
S_{k}(Q) \text { and } S_{k}\left(N u l l_{i}\right)\end{array}$ \\
\hline 7 & - & $\begin{array}{l}\text { Identify the } S_{k}(Q) \text { and } S_{k}\left(\mathrm{Null}_{i}\right) \text { with } \\
\text { the minimum } P \text {-value and define } \\
\text { these } P \text {-values as } P_{\min }(Q) \text { and } P_{\min }\left(N u l_{i}\right)\end{array}$ \\
\hline 8 & - & $\begin{array}{l}\text { Estimate the overall significance of } \\
P_{\min }(Q) \text { as the proportion of } V(\text { Null }) \text { vectors } \\
\text { that have a } P_{\min }(\text { Null }) \leq P_{\min }(Q)\end{array}$ \\
\hline
\end{tabular}

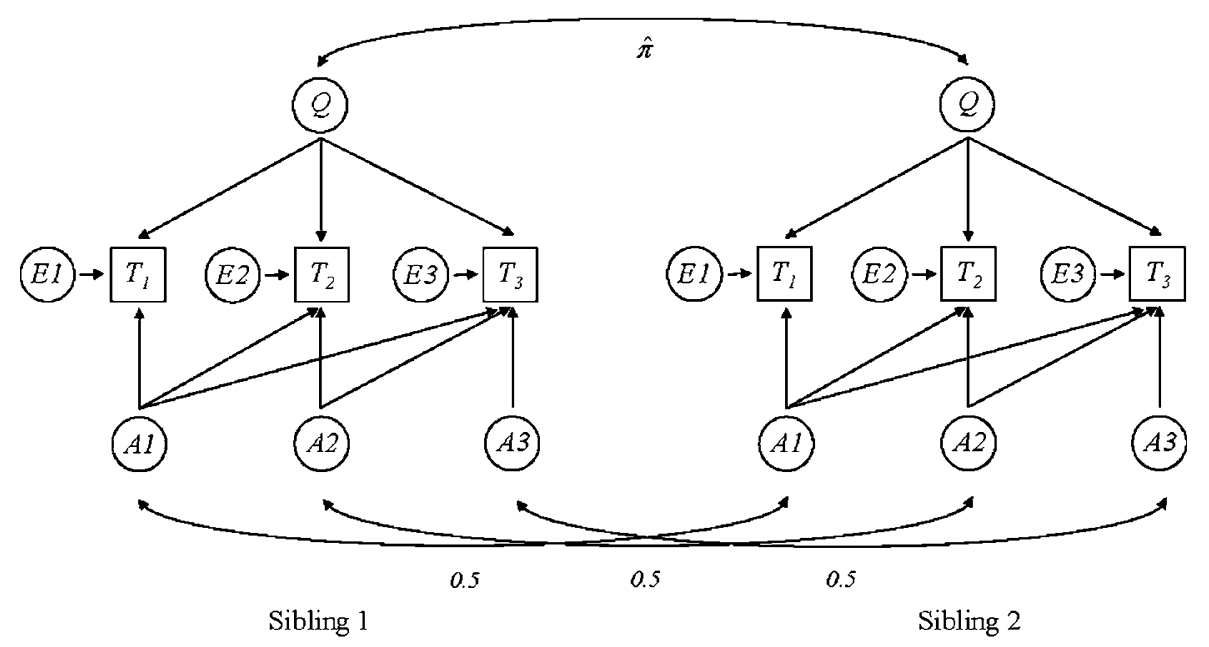

Figure 1 Multivariate model used to simulate three phenotypes $\left(T_{1}-T_{3}\right)$ in 250 sib-pairs. We assumed no environmental covariance between traits. Thus, the influence of $E 1$ on traits 2 and 3 and of $E 2$ on trait 3 was fixed at zero and, for simplicity, the corresponding paths are not shown in this figure. $\hat{\pi}$, proportion of alleles shared identical by descent at locus $Q$. $A 1-A 3$, latent residual additive genetic effects, correlated 0.5 between siblings. $E 1-E 3$, environmental effects not shared between siblings.

log-likelihood of the data when the variance of each trait was modelled to be explained by residual additive genetic effects $(A)$, environmental effects not shared between relatives $(E)$ and by the QTL effects $(Q)$, and $\ln \left(L_{A E}\right)$ is the natural log-likelihood of the data when the effect of $Q$ on the three traits was fixed at zero. The three test statistics obtained with the univariate analyses were inserted into a
$1 \times 3$ vector $V(Q)$ and analysed as described above. All univariate and multivariate test statistics were converted to empirical pointwise $P$-values using the 5000 replicates generated under models 9 (used for models 1-4) or 10 (for models 5-8). These replicates correspond to the $V(\mathrm{Null})$ vectors and were used for all subsequent permutation tests. 
Models 9 and 10 were also used to assess the experimentwise type-I error rate of the univariate analyses. The type-I error rate was estimated for an $\alpha=0.05$ as the proportion of replicates with a test statistic for at least one trait with an empirical pointwise $P$-value $\leq 0.05$. This was performed under three situations: first, using the three observed empirical pointwise $P$-values uncorrected for multiple trait testing. Second, after correcting the $P$-values with a Bonferroni adjustment. In this case, we tested different adjustments (eg $P$-value $\times 2.9$ ) until a correction was obtained that resulted in a type-I error close to the nominal level. Finally, after correcting the $P$-values with the Combined-correction approach.

\section{Application to genome-wide linkage analyses of three} asthma traits

The data set analysed herein includes 180 parents and 408 siblings from 201 Australian families with data available for three asthma continuous traits: forced expiratory volume in $1 \mathrm{~s}\left(\mathrm{FEV}_{1}\right)$, baseline airway obstruction as measured by $\mathrm{FEV}_{1}$ /forced vital capacity (FVC) and total serum immunoglobulin E (IgE) levels. This data set is described in detail elsewhere. ${ }^{10}$ Briefly, of 215 sib-pairs available for analysis, 12 (6\%) were genotyped at 201-300 markers, 104 (48\%) at 301-500 markers, 53 (25\%) at 501-1000 and $46(21 \%)$ at 1001-1544 markers. The three traits were normalised and adjusted for significant covariates (age, gender, height $\left(\mathrm{FEV}_{1}\right)$ and ever smoking $\left(\mathrm{FEV}_{1} / \mathrm{FVC}\right)$ ).

Univariate and multivariate $\mathrm{VC}$ analyses of the three traits were performed with SOLAR $3.0 .3^{14}$ with ascertainment correction. The univariate likelihood-ratio test statistics recorded for $\mathrm{FEV}_{1}, \mathrm{FEV}_{1} / \mathrm{FVC}$ and $\mathrm{IgE}$ with the real genome-scan at each of the 1796 positions analysed throughout the 22 autosomes $(3548 \mathrm{cM}$ at $2 \mathrm{cM}$ interval) were inserted into a $1 \times 3 V(Q)$ vector. Each of these $V(Q)$ vectors was then analysed as described above. In this case, we used MERLIN $0.10 .1^{15}$ to generate 1000 genome-scan replicates that retained the original phenotypes but with new genotypes simulated under the null hypothesis of no linkage for all autosomes, preserving the same allele frequencies, missing data pattern and marker spacing. The resulting 1796000 marker replicates were analysed with the three traits and used to assess empirical pointwise and genome-wide significance.

For the multivariate VC analysis, we used the unconstrained multivariate test implemented in SOLAR, which estimates six QTL parameters, three covariances and three variances. The test for linkage consists of fixing to zero all six parameters and then compare the likelihood of the data under this model with the likelihood of the data when the six parameters are estimated. As noted by others, ${ }^{8}$ we found that this test provides similar empirical power to detect a pleiotropic QTL as the constrained test that fixes the QTL correlations to 1 (equivalent to the model fitted in $\mathrm{Mx}$ for the analysis of the simulated datasets). However, the unconstrained test is more appropriate when the pattern of QTL genotypic effects may differ between traits. The pointwise significance of the multivariate likelihoodratio test statistic was estimated through the analysis of 15000 marker replicates simulated under the null hypothesis of no QTL effects on any trait.

\section{Results \\ Combined-correction: robust adjustment for multiple trait testing}

We first estimated the experiment-wise type-I error obtained with different univariate methods when multiple traits have been tested (Table 2). As expected, when considering the uncorrected $P$-values recorded with the three univariate analyses, the type-I error was highly inflated $(\sim 14 \%$ for $\alpha=0.05)$. Correcting the $P$-value of each trait with a Bonferroni adjustment resulted in a correct type-I error only if the appropriate adjustment was used $(P$-value $\times 2.9$ for a trait correlation of 0.25 and $\times 2.8$ for 0.50$)$. The experiment-wise type-I error of the Combined-correction approach was close to the nominal level with the advantage that no assumptions were made about the number of independent tests performed. As this method provided identical power to detect linkage as the appropriate Bonferroni approach (not shown), the Combined-correction approach is an efficient and robust alternative to estimate the evidence for linkage between one trait and one marker when multiple traits have been tested.

\section{Combined-sum: efficient detection of pleiotropic QTL}

We then investigated the power of the Combined-sum approach to detect a pleiotropic QTL when compared to univariate and multivariate VC analysis (Table 3). First, when the QTL had an effect on only one trait, univariate VC, multivariate VC and the combined approach provided similar power when the trait correlation was low (0.25). However, when the trait correlation was high (0.50), there was a slight improvement in the power of multivariate VC, consistent with previous findings. ${ }^{16}$ Second, when the QTL had an effect on two of the three traits, multivariate VC provided greatest power, followed by the Combined-sum approach, which in turn performed slightly better than univariate VC. This held for both low and high trait correlations. Third, when the QTL influenced all three traits equally, multivariate VC outperformed the Combined-sum approach only when the trait correlation was low. When the traits had high residual correlations, which had the same sign as the trait correlation induced by the QTL, the power of multivariate VC was markedly reduced. This was not entirely surprising and reflects a known theoretical property of multivariate linkage methods. ${ }^{16,17}$ As a result, the Combined-sum approach 
outperformed both univariate and multivariate VC in the presence of a high trait correlation. Similar but less pronounced results were observed when the QTL had an uneven effect on all three traits.

Estimating the number of traits linked to a marker Finally, we investigated the accuracy of the Combinedsum approach to estimate the number of traits influenced by the pleiotropic QTL. For this purpose, we compared the number of traits identified as being linked to $Q$ (estimated by $\left.k_{\text {min }}(Q)\right)$ with the number of traits that were truly influenced by $Q$ (Table 4). The combined approach correctly identified the number of traits influenced by $Q$ for $58 \%$ of the data sets when $Q$ influenced one trait only, for $62 \%$ when it influenced two traits and for $50 \%$ when it

Table 2 Experiment-wise type-I error rates at $\alpha=0.05$ of univariate VC linkage analysis of three correlated traits before and after correction for multiple trait testing

\begin{tabular}{lcc}
\hline Method $^{\mathrm{a}}$ & \multicolumn{2}{c}{ Trait correlation } \\
& 0.25 & 0.50 \\
\hline Univariate VC & 0.1406 & 0.1370 \\
Bonferroni $\times 2.0$ & 0.0728 & 0.0700 \\
Bonferroni $\times 2.8$ & 0.0522 & 0.0500 \\
Bonferroni $\times 2.9$ & 0.0504 & 0.0484 \\
Bonferroni $\times 3.0$ & 0.0488 & 0.0466 \\
Combined-correction & 0.0498 & 0.0494 \\
\hline
\end{tabular}

${ }^{a}$ Type-I error rate was calculated using the three observed $P$-values uncorrected for multiple trait testing (univariate VC), after correcting the three $P$-values with different Bonferroni adjustments (eg $P$-value $\times 2.9$ ) and after correcting the three $P$-values with the Combinedcorrection approach. See Methods for details. influenced the three traits equally. Note that the proportion of misclassified data sets reflected to a great extent the finite size of the sample simulated, (250 sib-pairs) which, in some instances, resulted in data sets having traits with QTL heritabilities very different from the generating model. For example, the mean QTL heritability in the 47 data sets simulated under model 1 that had a $k_{\min }(Q)=3$ was 0.29 (range $0.10-0.43$ ) for trait $1,0.18$ (range $0.07-0.36$ ) for trait 2 and 0.21 (range $0.07-0.42$ ) for trait 3 . When this effect was taken into account, the resolution of the Combined-sum approach to identify the number of traits influenced by a QTL was more apparent (Figure 2). Thus, as widely documented for the power of linkage analysis, the accuracy of the number of traits estimated by the combined approach to be influenced by $Q$ will depend on the number of families tested and on the QTL effect size.

\section{Application to genome-wide linkage analysis of three asthma traits}

We first performed genome-wide linkage analysis of $\mathrm{FEV}_{1}$, $\mathrm{FEV}_{1} / \mathrm{FVC}$ and IgE using a multivariate $\mathrm{VC}$ test. Six regions exceeded an empirical pointwise $P$-value of 0.01 : chromosomes 1p36, 5q13, 12q24, 17p13, 17q25 and 20q13 (Figure $3 \mathrm{a}$ ). The best result was recorded near markers D12S97 and D12S343 of 12q24; the likelihood-ratio test statistic observed at position $164 \mathrm{cM}$ of chromosome 12 (24.986) was never matched or exceeded in the 15000 marker replicates simulated under the null hypothesis of no linkage (pointwise $P<0.00007)$. One or multiple QTL in this region explained $55 \pm 29 \%( \pm 95 \% \mathrm{CI})$ of the variation of $\mathrm{FEV}_{1}, 47 \pm 19 \%$ of $\mathrm{FEV}_{1} / \mathrm{FVC}$ and $41 \pm 28 \%$ of $\mathrm{IgE}$, but these estimates can have a large upward bias. ${ }^{18}$ The analysis of one complete genome-scan replicate took $\sim 15$ days to

Table 3 Power of univariate VC, multivariate VC and that of the Combined-sum approach to detect linkage to a QTL that influenced one or more traits

\begin{tabular}{|c|c|c|c|c|c|c|c|c|c|c|c|}
\hline \multirow{2}{*}{ Trait 1} & \multirow[t]{2}{*}{$\begin{array}{c}\text { QTL variance } \\
\text { Trait } 2\end{array}$} & \multirow{2}{*}{ Trait 3} & \multirow[t]{2}{*}{ Method } & \multicolumn{4}{|c|}{$\begin{array}{c}\text { Trait correlation }=0.25 \\
\text { Power } \text { when type I error }=\end{array}$} & \multicolumn{4}{|c|}{$\begin{array}{c}\text { Trait correlation }=0.50 \\
\text { Power when type I error }=\end{array}$} \\
\hline & & & & 0.05 & 0.01 & 0.005 & 0.001 & 0.05 & 0.01 & 0.005 & 0.001 \\
\hline 0.25 & 0 & 0 & $\begin{array}{l}\text { Univariate VCa } \\
\text { Multivariate VC } \\
\text { Combined-sum }\end{array}$ & $\begin{array}{l}0.358 \\
0.338 \\
0.363\end{array}$ & $\begin{array}{l}0.162 \\
0.143 \\
0.171\end{array}$ & $\begin{array}{l}0.124 \\
0.109 \\
0.103\end{array}$ & $\begin{array}{l}0.048 \\
0.032 \\
0.040\end{array}$ & $\begin{array}{l}0.347 \\
0.517 \\
0.335\end{array}$ & $\begin{array}{l}0.159 \\
0.228 \\
0.161\end{array}$ & $\begin{array}{l}0.110 \\
0.173 \\
0.104\end{array}$ & $\begin{array}{l}0.063 \\
0.051 \\
0.014\end{array}$ \\
\hline 0.25 & 0.25 & 0 & $\begin{array}{l}\text { Univariate VC } \\
\text { Multivariate VC } \\
\text { Combined-sum }\end{array}$ & $\begin{array}{l}0.563 \\
0.760 \\
0.626\end{array}$ & $\begin{array}{l}0.278 \\
0.524 \\
0.387\end{array}$ & $\begin{array}{l}0.190 \\
0.438 \\
0.272\end{array}$ & $\begin{array}{l}0.061 \\
0.236 \\
0.160\end{array}$ & $\begin{array}{l}0.541 \\
0.836 \\
0.583\end{array}$ & $\begin{array}{l}0.266 \\
0.586 \\
0.318\end{array}$ & $\begin{array}{l}0.177 \\
0.526 \\
0.221\end{array}$ & $\begin{array}{l}0.089 \\
0.267 \\
0.113\end{array}$ \\
\hline 0.25 & 0.25 & 0.25 & $\begin{array}{l}\text { Univariate VC } \\
\text { Multivariate VC } \\
\text { Combined-sum }\end{array}$ & $\begin{array}{l}0.695 \\
0.899 \\
0.814\end{array}$ & $\begin{array}{l}0.370 \\
0.739 \\
0.611\end{array}$ & $\begin{array}{l}0.246 \\
0.668 \\
0.492\end{array}$ & $\begin{array}{l}0.080 \\
0.426 \\
0.291\end{array}$ & $\begin{array}{l}0.709 \\
0.692 \\
0.816\end{array}$ & $\begin{array}{l}0.391 \\
0.405 \\
0.540\end{array}$ & $\begin{array}{l}0.272 \\
0.344 \\
0.459\end{array}$ & $\begin{array}{l}0.128 \\
0.139 \\
0.215\end{array}$ \\
\hline 0.25 & 0.12 & 0.06 & $\begin{array}{l}\text { Univariate VC } \\
\text { Multivariate VC } \\
\text { Combined-sum }\end{array}$ & $\begin{array}{l}0.434 \\
0.481 \\
0.492\end{array}$ & $\begin{array}{l}0.195 \\
0.220 \\
0.269\end{array}$ & $\begin{array}{l}0.139 \\
0.161 \\
0.163\end{array}$ & $\begin{array}{l}0.039 \\
0.056 \\
0.069\end{array}$ & $\begin{array}{l}0.416 \\
0.359 \\
0.464\end{array}$ & $\begin{array}{l}0.184 \\
0.145 \\
0.227\end{array}$ & $\begin{array}{l}0.133 \\
0.112 \\
0.158\end{array}$ & $\begin{array}{l}0.074 \\
0.023 \\
0.046\end{array}$ \\
\hline
\end{tabular}

a Power was estimated for univariate VC after correcting the $P$-values of the three traits for multiple trait testing with the Combined-correction approach. 
Table 4 Number of data sets identified by the Combined-sum approach as having one, two or three traits linked to the quantitative trait locus (QTL) simulated under models 1-4

\begin{tabular}{|c|c|c|c|c|}
\hline \multirow[t]{2}{*}{ Number of traits found to be linked to the $Q T L^{\mathrm{a}}$} & \multicolumn{4}{|c|}{ Model (QTL variance for traits 1, 2 and 3) } \\
\hline & $1(0.25,0,0)$ & $2(0.25,0.25,0)$ & $3(0.25,0.25,0.25)$ & $4(0.25,0.12,0.06)$ \\
\hline $\begin{array}{l}1 \\
2 \\
3\end{array}$ & $\begin{array}{r}210(58 \%) \\
106(29 \%) \\
47(13 \%)\end{array}$ & $\begin{array}{l}102(16 \%) \\
387(62 \%) \\
137(22 \%)\end{array}$ & $\begin{array}{c}40(5 \%) \\
363(45 \%) \\
411(50 \%)\end{array}$ & $\begin{array}{l}130(26 \%) \\
201(41 \%) \\
161(33 \%)\end{array}$ \\
\hline Total $^{\mathrm{b}}$ & 363 & 626 & 814 & 492 \\
\hline
\end{tabular}

${ }^{a}$ As estimated by $k_{\min }(Q)$. See Methods for details.

${ }^{\mathrm{b}}$ Total number of data sets (out of 1000 for each model) that showed evidence for linkage to the QTL with the Combined-sum approach (ie $\left.P_{\text {final }}(Q) \leq 0.05\right)$. Corresponds to the power reported in Table 3 for $\alpha=0.05$. Similar results were observed for the four models with increased genetic correlation (models $5,6,7$ and 8 ).

Note: In model 1, for $1 / 210(0.5 \%)$ data sets that correctly identified the number of traits influenced by the QTL, the traits found to be linked to the QTL were not trait 1. Similarly, in model 2, for 20/382 (5.2\%) data sets, the traits found to be linked to the QTL were not traits 1 and 2 . This reflects the finite sampling size used for the simulations (see text).
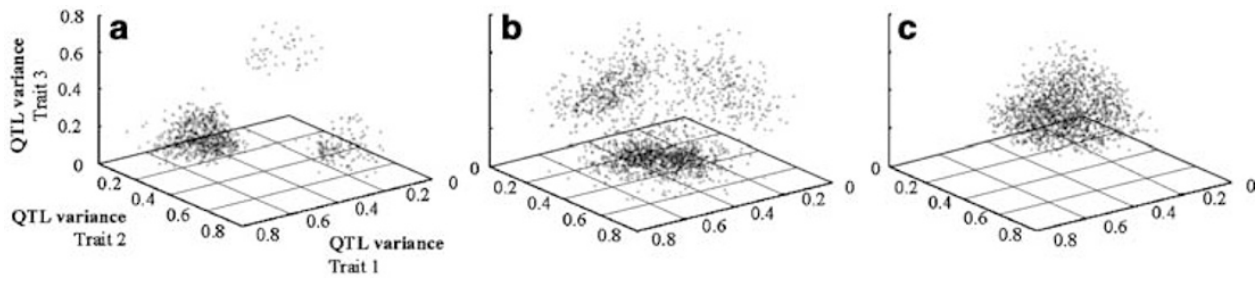

Figure 2 Data sets identified by the Combined-sum approach as having one (a), two (b) or three (c) traits influenced by a simulated QTL (Q), as a function of the proportion of variance of each trait explained by $Q$. Data sets from models 1 to 8 were considered that showed evidence for linkage to $Q$ with the combined approach, that is, $P_{\text {final }}(Q) \leq 0.05(N=4493)$. This total number of data sets is broken down into 363 from model 1,626 from model 2, 814 from model 3, 492 from model 4, 335 from model 5, 583 from model 6, 816 from model 7 and 464 from model 8 (cf. Table 3).

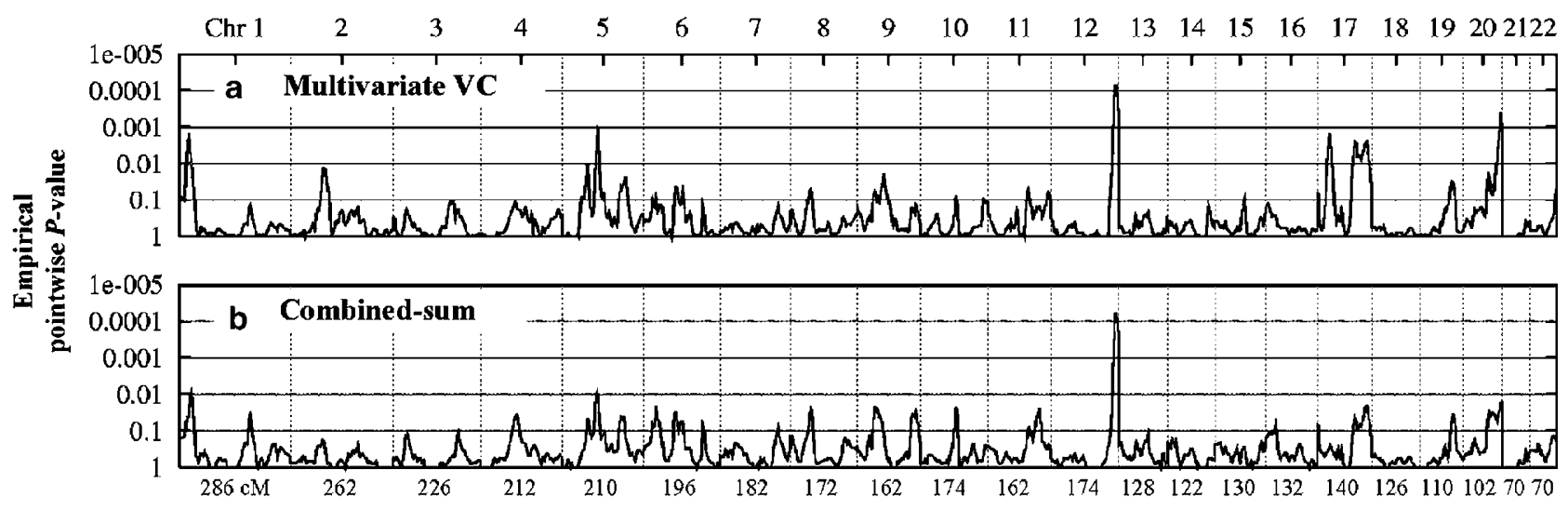

Figure 3 Genome-wide linkage analysis of $\mathrm{FEV}_{1}, \mathrm{FEV}_{1} / \mathrm{FVC}$ and IgE. For the multivariate variance components (VC) test (a), empirical pointwise $P$-values were estimated from 15000 marker replicates simulated under the null hypothesis of no linkage (for $12 q 24, P<1 / 15000$ ). For the Combined-sum test (b), 1000 complete genome-scan replicates were used to assess empirical significance (see Methods for details). Genetic distances are expressed in Kosambi centiMorgans (cM). The characteristics of the 408 siblings included for this analysis as well as univariate VC analysis of each trait are reported elsewhere. ${ }^{10}$ Heritabilities were $71+7 \%(+95 \% \mathrm{Cl})$ for $\mathrm{FEV}_{1}, 51+8 \%$ for $\mathrm{FEV}_{1} / \mathrm{FVC}$ and $52+9 \%$ for IgE. Genetic correlations were $0.33 \pm 0.12$ between $\mathrm{FEV}_{1}$ and $\mathrm{FEV}_{1} / \mathrm{FVC},-0.18 \pm 0.14$ between $\mathrm{FEV}_{1}$ and $\operatorname{lgE}$ and $-0.07 \pm 0.15$ between $\mathrm{FEV}_{1} / \mathrm{FVC}$ and IgE. The correlation between environmental factors not shared between relatives was $0.42 \pm 0.11$ for $\mathrm{FEV}_{1}$ and $\mathrm{FEV}_{1} / \mathrm{FVC}, 0.04 \pm 0.15$ for $\mathrm{FEV} \mathrm{F}_{1}$ and IgE and $-0.25+0.11$ for $\mathrm{FEV}_{1} / \mathrm{FVC}$ and IgE. Maximum-likelihood trait correlations were 0.35 (between $\mathrm{FEV}_{1}$ and $\left.\mathrm{FEV}_{1} / \mathrm{FVC}\right),-0.10\left(\mathrm{FEV}{ }_{1}\right.$ and $\left.\operatorname{lgE}\right)$ and $-0.16(\mathrm{FEV} / \mathrm{FVC}$ and $\lg \mathrm{E})$. 
Table 5 Summary of results from genome-wide linkage analysis of three asthma traits with univariate VC, multivariate VC and with the Combined-sum approach

\begin{tabular}{|c|c|c|c|c|c|c|}
\hline \multirow[t]{3}{*}{ Region $\left(C M^{\mathrm{a}}\right)$} & \multirow[t]{3}{*}{ Peak markers } & \multicolumn{5}{|c|}{ Empirical pointwise $P$-value $e^{\mathrm{b}}$} \\
\hline & & \multicolumn{3}{|c|}{ Univariate VC } & \multirow[t]{2}{*}{ Multivariate VC } & \multirow[t]{2}{*}{ Combined-sum $\left[k_{\min }(Q)^{c}\right]$} \\
\hline & & $\mathrm{FEV}_{1}$ & $F E V_{1} / F V C$ & $\lg E$ & & \\
\hline $5 q 13(90)$ & D5S424 & 0.00584 & 0.17365 & 0.51659 & 0.00107 & $0.00816[2]$ \\
\hline $12 q 24(164-166)$ & D12S97, D12S343 & 0.00489 & 0.00089 & 0.06374 & $<0.00007$ & $0.00006[3]$ \\
\hline $17 p 13(32-34)$ & D17S1852, D17S1303 & 0.36521 & 0.36026 & 1.00000 & 0.00147 & $0.24889[2]$ \\
\hline $17 q 25(128)$ & D17S784 & 0.01330 & 0.33888 & 0.66338 & 0.00253 & $0.02047[1]$ \\
\hline
\end{tabular}

$\mathrm{FEV}_{1}$, baseline forced expiratory volume in $1 \mathrm{~s} ; \mathrm{FEV}_{1} / \mathrm{FVC}$, ratio of $\mathrm{FEV}_{1}$ and baseline forced vital capacity; lgE, total serum immunoglobulin $\mathrm{E}$ levels. ${ }^{\mathrm{a}}$ Marker locations are given in Kosambi $\mathrm{cM}$ and have been interpolated from published physical positions and genetic maps. ${ }^{29}$

${ }^{\mathrm{b}}$ Empirical pointwise $P$-values were estimated for univariate VC and the Combined-sum analyses from 1000 genome-scan replicates simulated under the null hypothesis of no linkage (see Methods for details). For univariate VC, $P$-values were corrected for multiple trait testing with the Combinedcorrection approach. For the multivariate analysis, $P$-values were estimated from 15000 marker replicates simulated under the null hypothesis of no linkage to any of the three traits.

${ }^{c} k_{\text {min }}(Q)$, number of traits estimated to be influenced by one or more QTL linked to the peak marker(s).

run on a single $2.80 \mathrm{GHz} \mathrm{CPU}$, and, thus, genome-wide significance could not be estimated.

We then examined if some of these six regions could also be identified by the Combined-sum approach. In this case, one genome-scan replicate took $\sim 4 \mathrm{~h}$ to be analysed for all three traits $(\sim 90 \times$ faster $)$, and thus the overall analysis of the 1000 genome-scan replicates lasted $\sim 42$ days using four $2.80 \mathrm{GHz}$ CPUs. Results from the combined approach were comparable to the multivariate test (Figure $3 b$ ). Chromosomes 1p36, 5q13 and 12q24 were identified at the 0.01 significance level and chromosomes 17q25 and $20 q 13$ at the 0.05 level (Table 5). The best result was again recorded at $12 \mathrm{q} 24$ (pointwise $P=0.00006$ ). At this position, the $S_{k_{\text {min }}}(Q)$ statistic was observed when $k_{\text {min }}(Q)=3$ and the significance of this sum statistic was only matched or exceeded in one out of 1000 genome-scan replicates (genome-wide $P=0.001$ ). Had we used the best univariate $\mathrm{VC}$ result to assess linkage to this region (pointwise $P=0.00089$, for $\left.\mathrm{FEV}_{1} / \mathrm{FVC}\right)$, genome-wide significance would not have been reached (genome-wide $P=0.284$ ). Similarly, results from the Combined-sum approach suggest that chromosomes $1 \mathrm{p} 36\left(\mathrm{FEV}_{1}\right.$ and IgE, genomewide $P=0.166)$ and $5 \mathrm{q} 13\left(\mathrm{FEV}_{1}\right.$ and $\mathrm{FEV}_{1} / \mathrm{FVC}$, genomewide $P=0.167$ ) may also harbour a pleiotropic QTL associated with asthma susceptibility.

\section{Discussion}

The two methods described here are applicable for the empirical estimation of correct pointwise and genomewide significance when multiple traits are tested in the context of linkage studies. The crucial requirement for the application of both methods is that markers simulated under the null hypothesis of no linkage must be tested for linkage with all traits measured. In addition, if genomewide significance is to be estimated, complete genome-scan replicates must be simulated and analysed. Some studies already generate these analyses to estimate empirical significance and, in this case, the two approaches simply make additional use of that same data. On the other hand, for some studies, this may require a prohibitive amount of computer time, particularly if many traits are to be analysed with computationally intensive univariate methods such as maximum-likelihood VC linkage analysis. In this case, an alternative may be to use less-intensive nonparametric methods such as MERLIN-regress ${ }^{19}$ for continuous traits and allele sharing statistics for affection traits. $^{20}$

If the analysis of simulated data sets is not prohibitive, then the Combined-correction approach can be used to alleviate the acute problem of estimating linkage significance between one trait and one marker while accounting for multiple trait testing. This approach maintains the experiment-wise type-I error rate at nominal levels without requiring any assumptions to be made about the number of independent tests performed. It is applicable for any number of traits and for any trait correlation patterns.

With the Combined-sum approach, we hope to provide an efficient alternative to current multivariate linkage methods to detect regions that harbour pleiotropic QTL. Our simulations suggest that formal multivariate VC analysis is superior when compared to the Combinedsum approach in a number of situations. Therefore, it is the most appropriate method if it can be effectively applied. Additional advantages of the multivariate VC approach include the choice of fitting different nested linkage models that can be used to increase the power of the analysis (eg by constraining to 0 the QTL heritability of traits that show no evidence for linkage) and to formally test for pleiotropy. ${ }^{21}$ We did not test the power of such nested linkage models since it is not practical for genomewide scans to fit different multivariate models along the genome to account for the different linkage patterns observed at different markers. In addition, different tests 
have different distributions under the null hypothesis of no linkage, rending the assessment of significance difficult, and the number of tests performed would increase the experiment-wise type-I error rate.

In situations where multivariate VC is not feasible, an alternative may be to use the multivariate $\mathrm{HE}$ approach, ${ }^{9}$ a method that we did not test here. Nonetheless, this method is at present restricted to sib-pairs and it provides lower power when compared to multivariate VC. ${ }^{8}$

When multivariate methods are unfeasible or expected to be less powerful, we suggest that the Combined-sum approach can be used as a test to localise pleiotropic QTL. This approach may be particularly relevant when affection traits or a mixture of affection and continuous traits have been measured, when a large number of traits are to be analysed or when traits are moderately correlated and the QTL influences all traits. Unlike observed and previously described $^{6-8}$ for formal multivariate methods, the combined approach was mostly unaffected by the residual correlation between traits. For this reason, it may also prove useful for the analysis of longitudinal data, when strong environmental and genetic covariance between traits is expected.

A caveat of the Combined-sum approach is that if a region shows linkage to two or more traits, it is not possible to distinguish between the presence of a single QTL that influences multiple traits from the presence of multiple QTL each influencing a single trait. Nonetheless, not only this question may be beyond the scope of most linkage studies, as both situations may only be distinguishable with multivariate analysis of unrealistic large sample sizes, particularly in the presence of linkage disequilibrium. ${ }^{21,22}$

We exemplified the applicability of the Combined-sum approach to genome-wide linkage scans by combining univariate $\mathrm{VC}$ analyses of three asthma traits measured in 201 families and contrasting the results with a formal multivariate VC analysis. Results from both methods were comparable and identified chromosome 12q24 as a candidate region to harbour one or multiple QTL that influence(s) the variation of the two baseline lung function traits and of total serum IgE levels. The significance of linkage between these three traits and $12 \mathrm{q} 24$ reached genome-wide significance with the Combined-sum method but not when the best univariate result was considered. Genome-wide significance could not be assessed with the multivariate test. To date, chromosome 12 q24 has been reported to be linked to asthma traits in French, ${ }^{23}$ Dutch $^{24}$ and Japanese ${ }^{25}$ populations, but a major gene is yet to be cloned in this region. A number of association studies suggest that the nitric oxide synthase 1 gene is a likely candidate. ${ }^{26-28}$

In conclusion, our results confirm that the two methods described here can be used to combine results from multiple univariate linkage analyses for efficient, robust and powerful detection of human QTL. Further testing is required to assess how the Combined-sum approach performs under more specific scenarios, particularly with a large number of traits, with longitudinal data, with multiple linked QTL in incomplete linkage disequilibrium, and with a mixture of affection and continuous traits. It will also be important to investigate more thoroughly the behaviour of the test in the tail of its distribution and the situations when the combined approach outperforms multivariate VC.

\section{Acknowledgements}

We thank Carl Anderson, Allan McRae and David Smyth for the support to implement the simulations performed. MARF was supported by a doctorate grant from the Fundação para a Ciência e Tecnologia, Portugal.

\section{References}

1 Boomsma DI: Using multivariate genetic modeling to detect pleiotropic quantitative trait loci. Behav Genet 1996; 26: 161-166.

2 Eaves LJ, Neale MC, Maes H: Multivariate multipoint linkage analysis of quantitative trait loci. Behav Genet 1996; 26: 519-525.

3 de Andrade M, Thiel TJ, Yu L, Amos CI: Assessing linkage on chromosome 5 using components of variance approach: univariate versus multivariate. Genet Epidemiol 1997; 14: 773-778.

4 Blangero J, Almasy L: Multipoint oligogenic linkage analysis of quantitative traits. Genet Epidemiol 1997; 14: 959-964.

5 Martin N, Boomsma D, Machin G: A twin-pronged attack on complex traits. Nat Genet 1997; 17: 387-392.

6 Allison DB, Thiel B, St Jean P, Elston RC, Infante MC, Schork NJ: Multiple phenotype modeling in gene-mapping studies of quantitative traits: power advantages. Am J Hum Genet 1998; 63: $1190-1201$.

7 Evans DM, Duffy DL: A simulation study concerning the effect of varying the residual phenotypic correlation on the power of bivariate quantitative trait loci linkage analysis. Behav Genet 2004; 34: $135-141$.

8 Amos C, de Andrade M, Zhu D: Comparison of multivariate tests for genetic linkage. Hum Hered 2001; 51: 133-144.

9 Amos CI, Elston RC, Bonney GE, Keats BJ, Berenson GS: A multivariate method for detecting genetic linkage, with application to a pedigree with an adverse lipoprotein phenotype. Am J Hum Genet 1990; 47: 247-254.

10 Ferreira MAR, O'Gorman L, Le Souef P et al: Robust estimation of experiment-wise $P$-values applied to a genome-scan of multiple asthma traits identifies a new region of significant linkage on chromosome 20q13. Am J Hum Genet 2005; 77: 1075-1085.

11 Hoh J, Wille A, Ott J: Trimming, weighting, and grouping SNPs in human case-control association studies. Genome Res 2001; 11: $2115-2119$.

12 Dudbridge F, Koeleman BP: Efficient computation of significance levels for multiple associations in large studies of correlated data, including genomewide association studies. Am J Hum Genet 2004, 75: 424-435.

13 Neale M, Boker S, Xie G, Maes H: Mx: Statistical Modeling. Richmond, VA: Department of Psychiatry, 2002.

14 Almasy L, Blangero J: Multipoint quantitative-trait linkage analysis in general pedigrees. Am J Hum Genet 1998; 62: $1198-1211$

15 Abecasis GR, Cherny SS, Cookson WO, Cardon LR: Merlin - rapid analysis of dense genetic maps using sparse gene flow trees. Nat Genet 2002; 30: 97-101.

16 Jiang C, Zeng ZB: Multiple trait analysis of genetic mapping for quantitative trait loci. Genetics 1995; 140: 1111-1127. 
17 Korol AB, Ronin YI, Kirzhner VM: Interval mapping of quantitative trait loci employing correlated trait complexes. Genetics 1995; 140: 1137-1147.

18 Goring HH, Terwilliger JD, Blangero J: Large upward bias in estimation of locus-specific effects from genomewide scans. Am J Hum Genet 2001; 69: 1357-1369.

19 Sham PC, Purcell S, Cherny SS, Abecasis GR: Powerful regressionbased quantitative-trait linkage analysis of general pedigrees. Am J Hum Genet 2002; 71: 238-253.

20 Whittemore AS, Halpern J: A class of tests for linkage using affected pedigree members. Biometrics 1994; 50: 118-127.

21 Almasy L, Dyer TD, Blangero J: Bivariate quantitative trait linkage analysis: pleiotropy versus co-incident linkages. Genet Epidemiol 1997; 14: 953-958.

22 Knott SA, Haley CS: Multitrait least squares for quantitative trait loci detection. Genetics 2000; 156: 899-911.

23 Dizier MH, Besse-Schmittler C, Guilloud-Bataille M et al: Genome screen for asthma and related phenotypes in the French EGEA study. Am J Respir Crit Care Med 2000; 162: 1812-1818.
$24 \mathrm{Xu} \mathrm{J}$, Postma DS, Howard TD et al: Major genes regulating total serum immunoglobulin $\mathrm{E}$ levels in families with asthma. $\mathrm{Am} J$ Hum Genet 2000; 67: 1163-1173.

25 Yokouchi Y, Shibasaki M, Noguchi E et al: A genome-wide linkage analysis of orchard grass-sensitive childhood seasonal allergic rhinitis in Japanese families. Genes Immun 2002; 3: $9-13$.

26 Grasemann H, Yandava CN, Drazen JM: Neuronal NO synthase (NOS1) is a major candidate gene for asthma. Clin Exp Allergy 1999; 29 (Suppl 4): 39-41.

27 Shao C, Suzuki Y, Kamada F et al: Linkage and association of childhood asthma with the chromosome 12 genes. J Hum Genet 2004; 49: 115-122.

28 Wechsler ME, Grasemann H, Deykin A et al: Exhaled nitric oxide in patients with asthma: association with NOS1 genotype. Am J Respir Crit Care Med 2000; 162: 2043-2047.

29 Kong X, Murphy K, Raj T, He C, White PS, Matise TC: A combined linkage-physical map of the human genome. Am J Hum Genet 2004; 75: 1143-1148. 